\title{
Identification and integrated analysis of differentially expressed IncRNAs and circRNAs reveal the potential ceRNA networks during PDLSC osteogenic differentiation
}

\author{
Xiuge $\mathrm{Gu}^{1,2}$, Mengying $\mathrm{Li}^{1,2}$, Ye Jin ${ }^{1,2}$, Dongxu Liu ${ }^{1,2^{*}}$ and Fulan Wei ${ }^{1,2^{*}}$ (D)
}

\begin{abstract}
Background: Researchers have been exploring the molecular mechanisms underlying the control of periodontal ligament stem cell (PDLSC) osteogenic differentiation. Recently, long noncoding RNAs (IncRNAs) and circular RNAs (circRNAs) were shown to function as competitive endogenous RNAs (ceRNAs) to regulate the effect of microRNAs (miRNAs) on their target genes during cell differentiation. However, comprehensive identification and integrated analysis of IncRNAs and circRNAs acting as ceRNAs during PDLSC osteogenic differentiation have not been performed.

Results: PDLSCs were derived from healthy human periodontal ligament and cultured separately with osteogenic induction and normal media for 7 days. Cultured PDLSCs were positive for STRO-1 and CD146 and negative for CD31 and CD45. Osteo-induced PDLSCs showed increased ALP (alkaline phosphatase) activity and up-regulated expression levels of the osteogenesis-related markers ALP, Runt-related transcription factor 2 and osteocalcin. Then, a total of 960 IncRNAs and 1456 circRNAs were found to be differentially expressed by RNA sequencing. The expression profiles of eight IncRNAs and eight circRNAs were measured with quantitative real-time polymerase chain reaction and were shown to agree with the RNA-seq results. Furthermore, the potential functions of IncRNAs and circRNAs as ceRNAs were predicted based on miRanda and were investigated using Gene Ontology and Kyoto Encyclopedia of Genes and Genomes analysis. In total, 147 IncRNAs and 1382 circRNAs were predicted to combine with 148 common miRNAs and compete for miRNA binding sites with 744 messenger RNAs. These mRNAs were predicted to significantly participate in osteoblast differentiation, the MAPK pathway, the Wnt pathway and the signaling pathways regulating pluripotency of stem cells. Among them, IncRNAs coded as TCONS_00212979 and TCONS_00212984, as well as circRNA BANP and circRNA ITCH, might interact with miRNA34a and miRNA146a to regulate PDLSC osteogenic differentiation via the MAPK pathway.
\end{abstract}

Conclusions: This study comprehensively identified IncRNAs/circRNAs and first integrated their potential ceRNA function during PDLSC osteogenic differentiation. These findings suggest that specific IncRNAs and circRNAs might function as ceRNAs to promote PDLSC osteogenic differentiation and periodontal regeneration.

Keywords: Long non-coding RNAs, Circular RNA, Competitive endogenous RNA, Periodontal ligament stem cells, Osteogenic differentiation, RNA sequencing

\footnotetext{
* Correspondence: liudongxu@sdu.edu.cn; weifl@sdu.edu.cn

'Department of Orthodontics, Shandong Provincial Key Laboratory of Oral

Tissue Regeneration, School of Stomatology, Shandong University, Wenhua

Xi Road No. 44-1, Jinan, Shandong 250012, People's Republic of China

Full list of author information is available at the end of the article
} 


\section{Background}

Periodontitis is a slowly progressive disease characterized by the loss of periodontal tissue, which is the main cause of tooth loss [1]. With the development of stem-cell delivery therapeutics, periodontal ligament stem cells (PDLSCs) have been shown to produce typical periodontal ligament-like tissue to regenerate tissue damaged by periodontitis [2, 3]. Our previous studies also have shown the great potential of PDLSCs to regenerate periodontal tissue and form a bioengineered tooth root in miniature pigs [4-6]. The regeneration potency of PDLSCs contributes to their self-renewal and multi-differentiation capacity, especially osteogenic differentiation [7]. Exploring the molecular mechanisms of PDLSC osteogenic differentiation might provide new genetic strategies for periodontal regenerative medicine.

Recently, through microarray analysis, microRNAs (miRNAs) were identified and predicted to be involved in PDLSC osteogenic differentiation [8]. In addition, miRNA146a, miRNA17 and miRNA22 have been demonstrated to regulate PDLSC osteogenic differentiation by modulating the expression of target genes at the post-transcriptional level [9-11]. However, recent studies have revealed that a new player, competing endogenous RNA (ceRNA), is essential for the circuitry of miRNAs and target genes [12]. By competing for common miRNA response elements (MREs), ceRNAs can break the balance between miRNAs and target genes to regulate the physiological and pathophysiological process [13]. These ceRNAs include various types of RNAs, such as long non-coding RNAs (lncRNAs), circular RNAs (circRNAs), messenger RNAs (mRNAs) and pseudogenes.

LncRNA is a class of non-coding RNA (ncRNA) transcripts longer than 200 nucleotides [14]. Recent studies have reported that lncRNAs were involved in the osteogenic differentiation process [15]. For example, up-regulation of lncRNA HIF $1 \alpha$-anti-sense 1 induced by TGF $\beta$-mediated targeting of sirtuin 1 promotes the osteogenic differentiation of human bone marrow stromal cells [16]. Furthermore, lncRNA ANCR was proved to be critical in regulating the PDLSC osteogenic differentiation via the Wnt signaling pathway [17].

CircRNA is another new class of RNA composed of more than one exon with a covalently closed loop [18]. Compared to linear RNA, this circular structure is more stable and resistant to RNase $\mathrm{R}$ [19]. Emerging evidence has revealed that circRNAs participate in osteogenic differentiation [20, 21]. For instance, 154 differentially expressed circRNAs were found to associate with bone morphogenetic protein 2-induced osteogenic differentiation of MC3T3-E1 cells [22]. Moreover, circRNAs were predicted to have potential roles in osteogenesis of PDLSCs [23].

Although several lncRNAs, circRNAs and miRNAs are suggested to be involved in PDLSC osteogenic differentiation, there is not much data published on their potential networks and functions. To fully understand the impact of ceRNA crosstalk on PDLSC osteogenic differentiation, it will be crucial to integrate the IncRNA/circRNA-miRNA-mRNA competitive regulatory networks. In this study, we developed RNA sequencing (RNA-seq) with Illumina HiSeq2000 to comprehensively identify differentially expressed lncRNAs and circRNAs in normal and osteogenic inductive PDLSCs. Subsequently, the representative lncRNAs and circRNAs were further confirmed using quantitative real-time polymerase chain reaction (qRT-PCR). Finally, we predicted the ceRNA networks of lncRNAs, circRNAs, miRNAs and mRNAs based on miRanda and investigated their potential regulatory roles via gene ontology $(\mathrm{GO})$ and Kyoto Encyclopedia of Genes and Genomes (KEGG) analysis. Our findings might provide new evidence for exploring the molecular mechanism of PDLSC osteogenic differentiation.

\section{Methods}

All protocols for the handling of human tissues were approved by the Research Ethics Committee of Stomatology Hospital of Shandong University, China (G201401601). Informed consent was obtained from all donors.

\section{Cell culture and osteogenic induction}

In this study, PDLSCs were derived from the middle third of the root surface of normal human impacted third molars, which were collected from 18- to 20-yearold patients at the Department of Oral Maxillofacial Surgery, Stomatology Hospital of Shandong University, using the explant culture method. Then, they were cultured with normal media, consisting of $\alpha$-modification of Eagle's media (HyClone, South Logan, UT, USA), 10\% fetal calf serum (Gibco BRL, Grand Island, NY, USA), 100 U/ml penicillin and $100 \mu \mathrm{g} / \mathrm{ml}$ streptomycin (Invitrogen, Carlsbad, CA, USA) at $37{ }^{\circ} \mathrm{C}$ in $5 \%$ carbon dioxide. The stemness of PDLSCs was characterized by the scanning of cell surface markers (STRO-1, CD146, CD31, and CD45) through flow cytometric analysis (Becton, Dickinson and Company, Franklin Lakes, NJ, USA). For osteogenic differentiation, PDLSCs were cultured with osteogenic inductive media supplemented with $10 \mathrm{nM}$ dexamethasone, $10 \mathrm{mM} \beta$-glycerophosphate and $50 \mu \mathrm{g} / \mathrm{ml}$ vitamin C (Sigma-Aldrich, St. Louis, MO, USA). Through separately culture with osteogenic induction and normal media for 7 days, PDLSCs were divided into two groups: induced and non-induced groups. All cells in this study were used at passage number 3 . 


\section{Alkaline Phosphatase (ALP) staining and ALP activity assay}

ALP staining and activity assay were performed using an Alkaline Phosphatase Kit (Sigma-Aldrich) as described previously [24]. Briefly, PDLSCs were fixed with 70\% ethanol for $30 \mathrm{~min}$ and stained with a solution of sodium nitrite, FRV alkaline and naphthol AS-BI alkaline for 15 min away from light. For the ALP activity assays, total protein was isolated from two groups of PDLSCs, incubated for 15 min with citrate buffer and phosphatase substrate (Sigma-Aldrich), and then quantified by spectrophotometric absorbance at $405 \mathrm{~nm}$.

\section{Quantitative real-time PCR (qRT-PCR)}

Total RNA was isolated from two groups of PDLSCs. For analysis of linear transcripts, $1 \mu \mathrm{g}$ of RNA per sample was reverse transcribed into cDNA using a cDNA Reverse Transcription Kit (Takara, Tokyo, Japan).

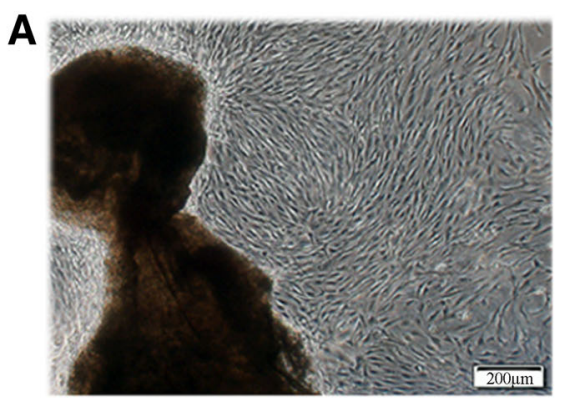

B

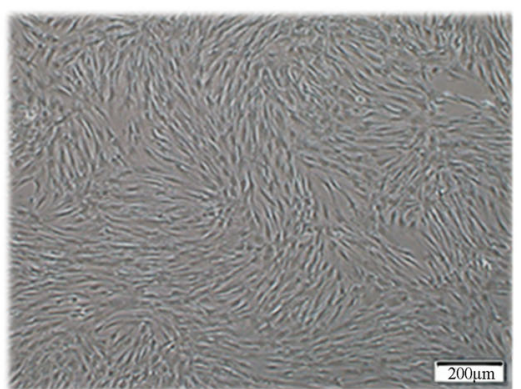

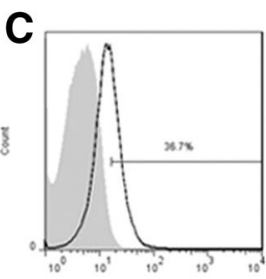

STRO-1

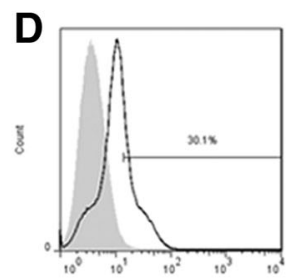

CD146 PE

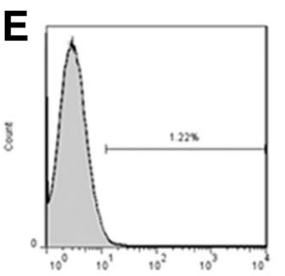

CD31 FITC

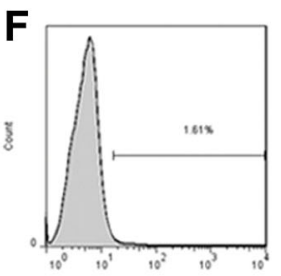

CD45 PE Cy5.5
G
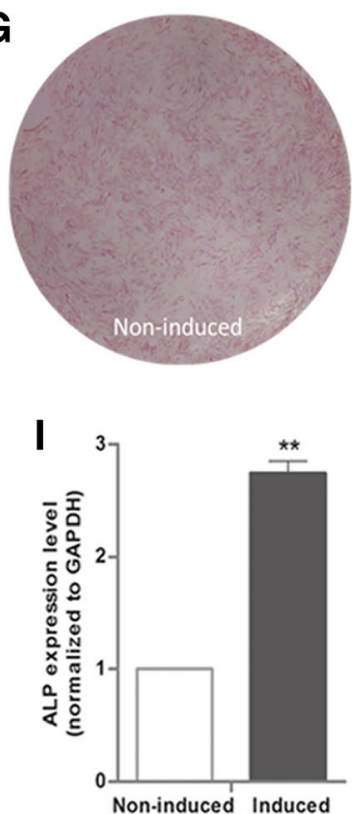
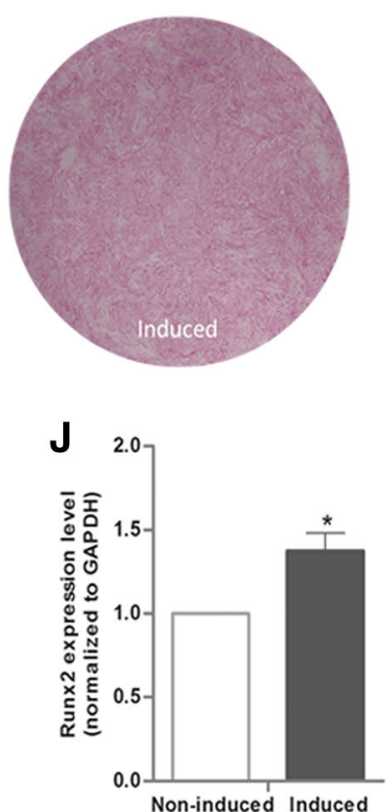

H
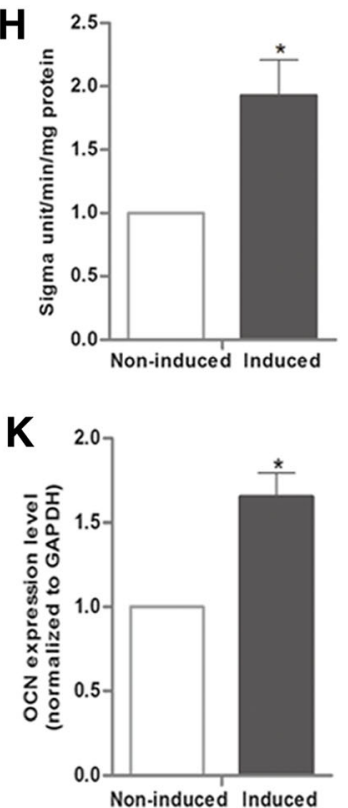

Fig. 1 Identification and osteogenic differentiation of PDLSCS. a PDLSCs were derived from periodontal ligament explants. b PDLSCs were cultured with non-induced media at passage number 3. c-f PDLSCs were positive for STRO-1 and CD146 and negative for CD31 and CD45. g, h ALP activity was enhanced in osteo-induced PDLSCs (Induced), as evidenced by ALP staining and ALP activity assay. i-k Compared with the non-induced group, the induced group showed up-regulated expression of the osteogenic genes ALP, Runx2 and OCN by qRT-PCR. All PCRs were performed in triplicate. The data are represented as means $\pm \mathrm{SD} .{ }^{*}, P<0.05 ;{ }^{*}, P<0.01$ 

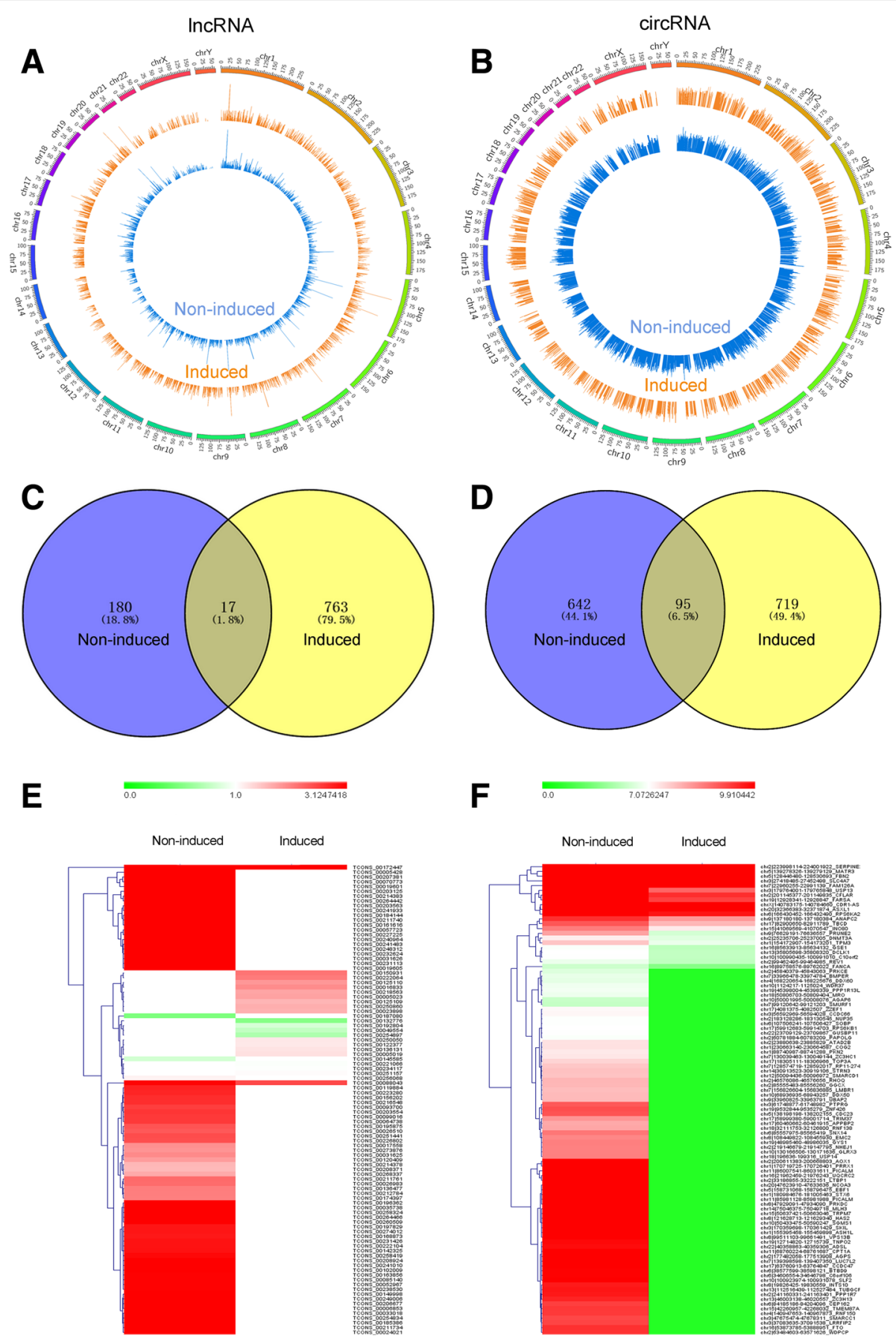

Fig. 2 The apparent variations of differentially expressed IncRNAs and circRNAs. (a, $\mathbf{b})$ LncRNAs and circRNAs were broadly distributed across the 24 pairs of human chromosomes according to their locations. The inner blue ring corresponds to the non-induced group; the outer yellow ring corresponds to the induced group. c Among differentially expressed IncRNAs, 17 common IncRNAs and 180 specific IncRNAs in the non-induced group and 763 specific IncRNAs in the induced group were identified. d Among differentially expressed circRNAs, 95 common circRNAs and 642 specific circRNAs in the non-induced group and 719 specific circRNAs in the induced group were identified. e Differentially expressed IncRNAs, consisting of 777 up-regulated IncRNAs and 183 down-regulated IncRNAs, are displayed in the heatmap. f Differentially expressed circRNAs, consisting of 766 up-regulated circRNAs and 690 down-regulated circRNAs, are displayed in the heatmap

Convergent primers were designed to detect lncRNAs and mRNAs. Divergent primers were designed to detect the circular form of circRNAs. Relative transcript levels were measured with quantitative PCR using a Roche LightCycler 480 sequence detection system (Roche, Basel, Switzerland) following the manufacturer's protocol. Each 
Table 1 Statistical data of RNA-Seq reads for two samples

\begin{tabular}{llllll}
\hline Sample & $\begin{array}{l}\text { Raw } \\
\text { reads }\end{array}$ & $\begin{array}{l}\text { Clean } \\
\text { reads }\end{array}$ & $\begin{array}{l}\text { Unique } \\
\text { IncRNAs }\end{array}$ & $\begin{array}{l}\text { Unique } \\
\text { circRNAs }\end{array}$ & $\begin{array}{l}\text { Unique } \\
\text { mRNAs }\end{array}$ \\
\hline Non-induced & 91.4 million & 90.9 million & 11,529 & 5913 & 66,134 \\
Induced & 113.8 million & 112.4 million & 9511 & 3162 & 52,227 \\
\hline
\end{tabular}

$20 \mu \mathrm{l}$ reaction volume contained $10 \mu \mathrm{LYSB} \mathrm{SP}^{\circ}$ Premix Ex $\mathrm{Taq}^{\mathrm{Tm}}$ (Takara), $0.4 \mu \mathrm{l} 10 \mu \mathrm{M}$ forward primer $(0.4 \mu \mathrm{M}$ final), $0.4 \mu \mathrm{l} 10 \mu \mathrm{M}$ reverse primer $(0.4 \mu \mathrm{M}$ final), $200 \mathrm{ng}$ of template cDNA and DEPC-treated water. GAPDH was used as an internal control to quantify and normalize the results. The primer pairs are listed in Additional file 1. The specificity of the reaction was determined by detection of the Tms of the amplification products immediately after the last reaction cycle. The $2^{-\triangle \Delta C T}$ value was used for comparative quantitation. All qRT-PCRs were performed in triplicate.

\section{Construction of cDNA libraries and high-throughput sequencing}

Total RNA was extracted from two groups using Trizol (Invitrogen) according to the manufacturer's protocols. Strand-specific cDNA libraries were constructed following a previously described protocol [25] and were sequenced using an Illumina HiSeq2000 sequencer (LC Biotech, Hangzhou, China) by performing a paired-end run with a $100 \mathrm{bp}$ read length. The raw reads were processed by removing the adaptor reads and low-quality tags. All subsequent analyses were performed using clean reads.

\section{Identification of differentially expressed IncRNAs, circRNAs and mRNAs}

The clean reads from two cDNA libraries were mapped to the human genome sequence in GENCODE Release 19 using TopHat version 2.0.9 [26]. The transcripts were then assembled and annotated using Cufflinks [27]. According to the annotation of the human genome sequence, the known lncRNAs and mRNAs were identified. The coding potential of the remaining unknown transcripts was calculated with a coding potential calculator based on

Table 2 Expression profiles of IncRNAs validated by RNA-seq

\begin{tabular}{llll}
\hline Test_id. & Induced (FPKM) & Non-induced (FPKM) & Regulation \\
\hline TCONS_00019601 & 93.6452 & 0 & up \\
TCONS_00227764 & 830.447 & 47.6825 & up \\
TCONS_00085268 & 0 & 26.6246 & down \\
TCONS_00254538 & $18,683.1$ & 3962.3 & up \\
TCONS_00198784 & 9.25995 & 0.0230801 & up \\
TCONS_00136898 & 8.67356 & 0.0105046 & up \\
TCONS_00125934 & 0 & 4.65289 & down \\
TCONS_00115113 & 0 & 4.05314 & down \\
\hline
\end{tabular}

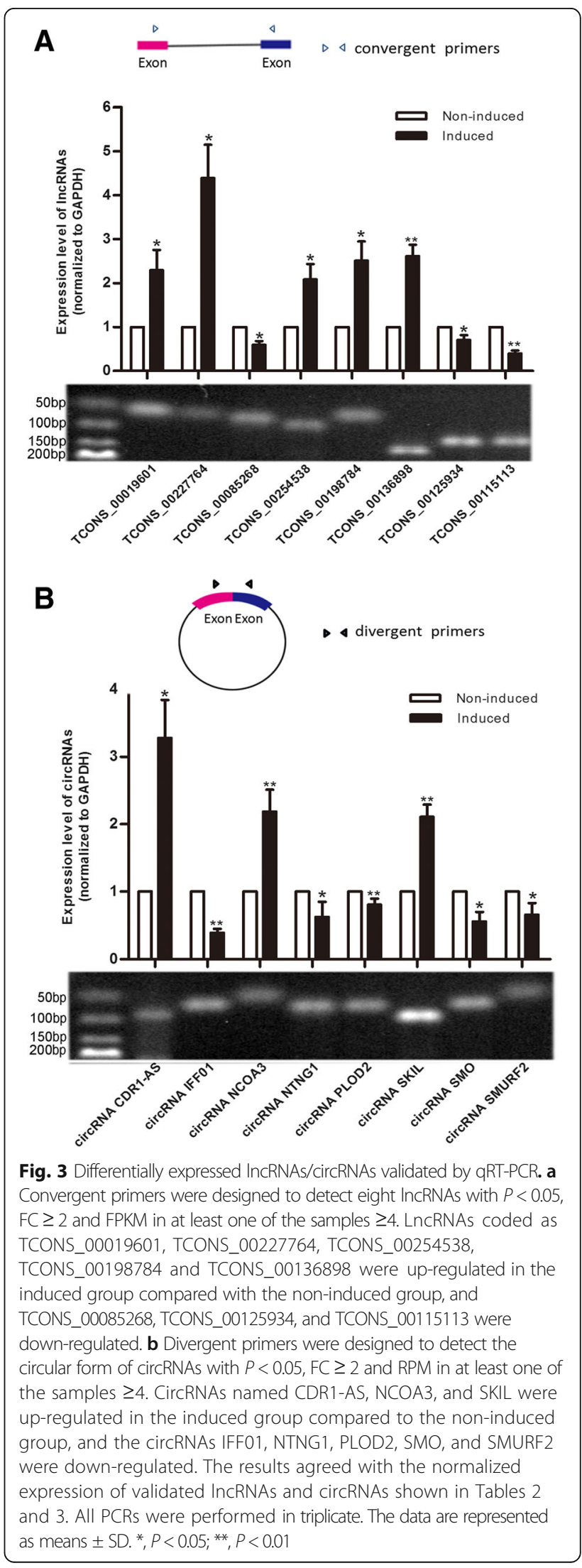


Table 3 Expression profiles of circRNAs validated by RNA-seq

\begin{tabular}{llll}
\hline Name & Induced (RPM) & Non-induced (RPM) & Regulation \\
\hline circRNA CDR1-AS & 3038.695 & 1102.345 & up \\
circRNA IFF01 & 0 & 337.2772 & down \\
circRNA NCOA3 & 1421.615 & 0 & up \\
circRNA NTNG1 & 0 & 1286.478 & down \\
circRNA PLOD2 & 0 & 3742.131 & down \\
circRNA SKIL & 1956.098 & 0 & up \\
circRNA SMO & 0 & 783.6154 & down \\
circRNA SMURF2 & 0 & 282.7267 & down \\
\hline
\end{tabular}

the quality, completeness, and sequence similarity of the open reading frame to the proteins in the protein databases [28]. The remaining unknown transcripts of more than 200 base pairs (bp) were considered novel lncRNAs with a coding potential score of less than -1 , which suggested that a transcript had no protein-coding capacity. The expression levels of lncRNAs and mRNAs were quantified as FPKM (fragments per kilobase of exon per million fragments mapped) using the Cuffdiff program [27]. The differential expression of lncRNAs and mRNAs was determined using DESeq, with $P<0.05$ and fold change (FC) $\geq 2$ [29].

Compared with linear RNAs, circRNAs exhibit distinct patterns of alternative back-splicing and alternative splicing. An upgraded computational pipeline (CIRCexplorer2) was used to systematically identify and annotate circRNAs [30]. The expression levels of circRNAs were quantified as RPM (mapped backsplicing junction reads per million mapped reads) using the EBSeq package [31]. The differential expression of circRNAs was determined with $P<0.05$, $\mathrm{FC} \geq 2$, and circRNA junction reads $\geq 5$ [31].

\section{Functional analysis}

The ceRNA networks among lncRNAs, circRNAs, miRNAs and mRNAs were predicted based on miRanda with a

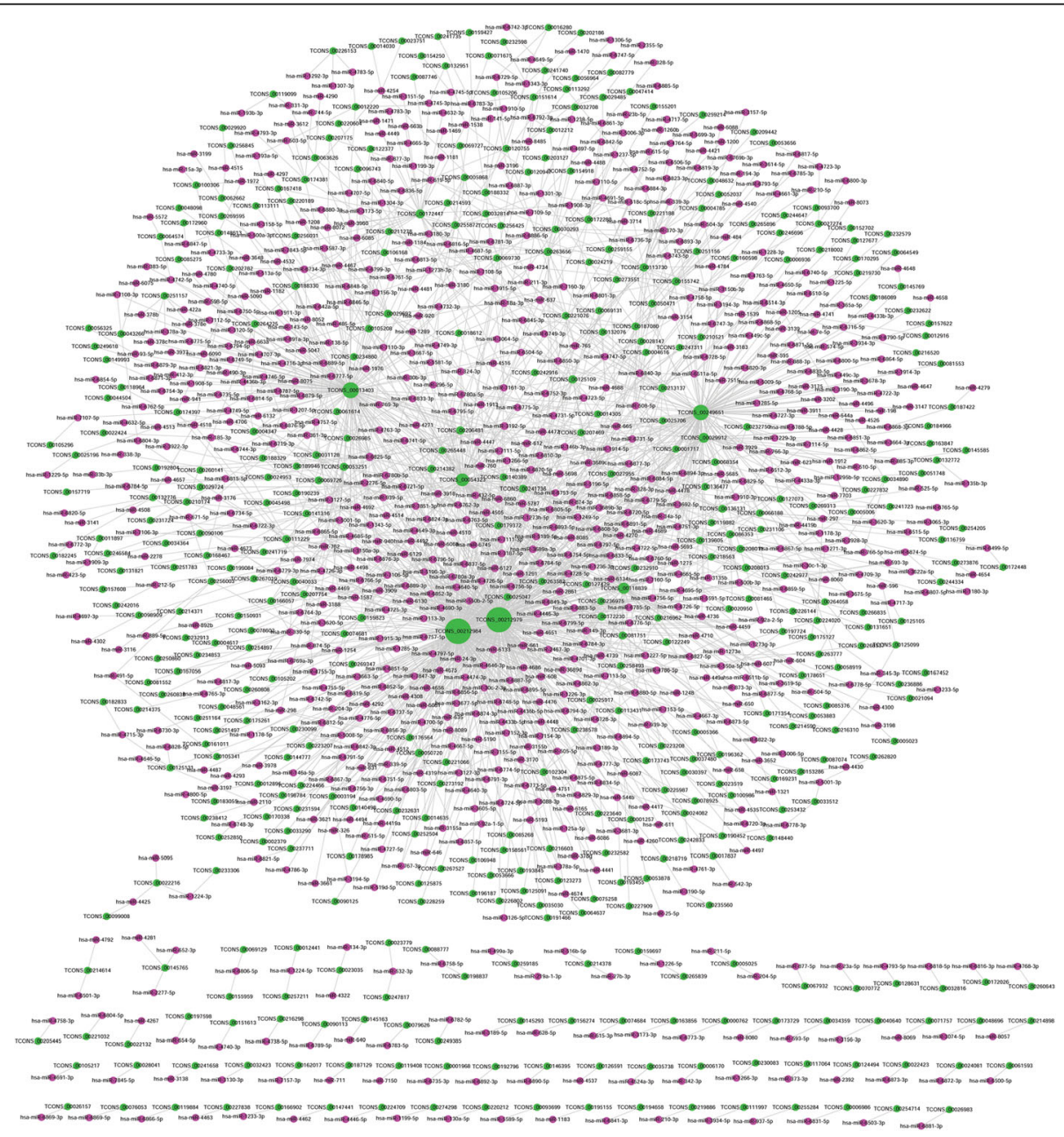

Fig. 4 CeRNA networks of 430 IncRNAs and 779 miRNAs based on miRanda 


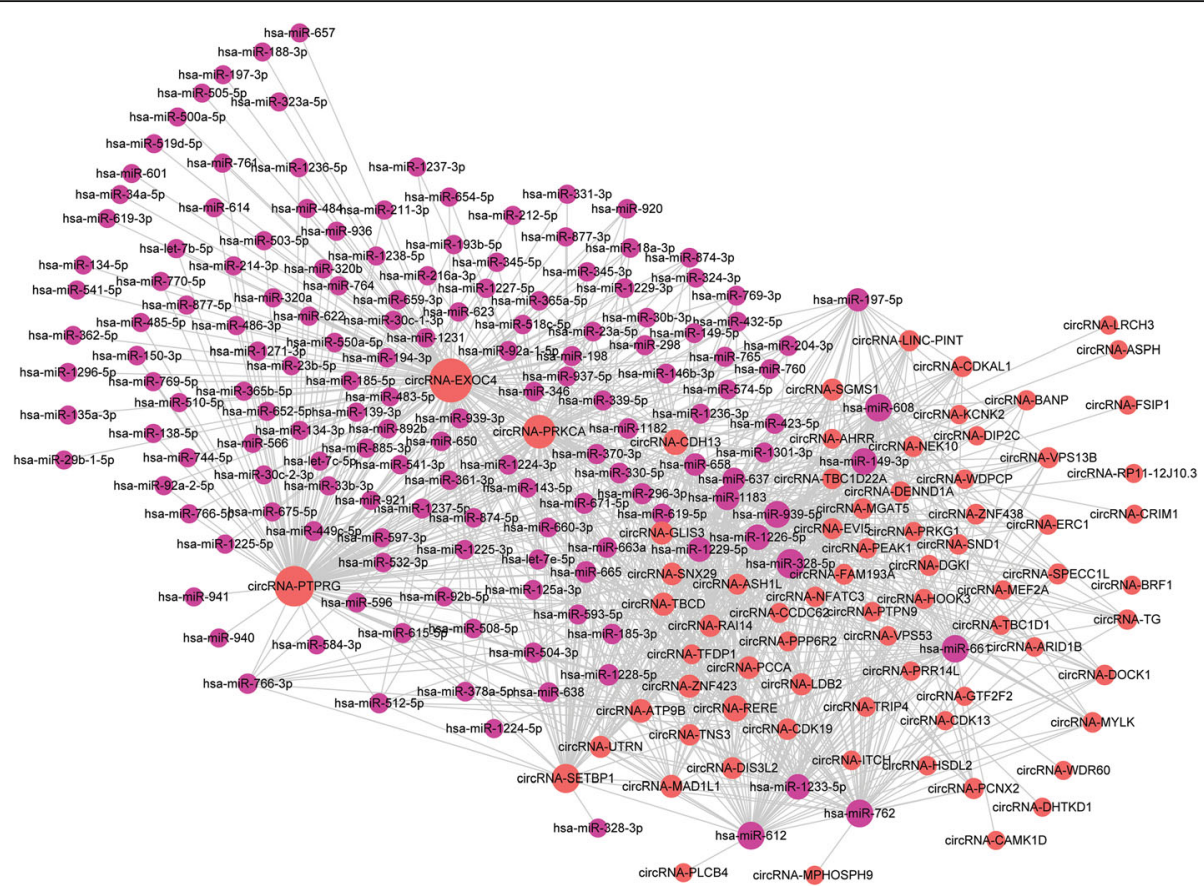

Fig. 5 CeRNA networks of selected circRNAs and miRNAs based on miRanda. Considering that graphics cannot display the enormous amount of network information between 1401 circRNAs and 855 miRNAs, we selected circRNAs with more miRNA binding sites and less binding free energy to make the network diagram using Cytoscape3.5.1

maximum binding free energy of -20 [32]. First, we predicted and selected miRNAs binding with both differentially expressed lncRNAs and circRNAs. Then, target mRNAs of these selected miRNAs were predicted and compared to the differentially expressed mRNAs that were identified in the RNA-seq results. Subsequently, we selected the intersecting elements of target mRNAs and differentially expressed mRNAs to analyze their potential functions through GO functional annotation and KEGG pathway analysis. GO terms were enriched using Blast2GO [33] by referring to GO databases. Meanwhile, KEGG pathway analysis was performed by referring to KEGG pathway databases. Cytoscape3.5.1 was used to display the lncRNA/circRNA-miRNA-mRNA networks.

\section{Statistical analysis}

Quantitative qRT-PCR datasets are presented as the means \pm standard deviation (SD) of at least three independent experiments. The statistical calculations were performed with SPSS statistics software version 17.0. Student's t-test was performed for normally distributed data to determine statistical significance. A $P$-value less than 0.05 was considered statistically significant.

\section{Results}

Identification and Osteogenic differentiation of PDLSCs PDLSCs derived from periodontal ligament explants were cultured with normal media to passage number 3
(Fig. 1a, b). Cultured PDLSCs were positive for STRO-1 and CD146 and negative for CD31 and CD45 (Fig. 1c-f). Increased ALP activity identified via ALP staining and ALP activity assay indicated osteogenic differentiation of osteo-induced PDLSCs (Fig. 1g, h). Subsequently, the up-regulated expression levels of the osteogenesisrelated markers ALP, Runt-related transcription factor 2 (Runx2) and osteocalcin (OCN) provided further proof for the occurrence of PDLSC osteogenic differentiation (Fig. 2i-k). These findings agreed with previous reports on PDLSC differentiation into osteoblasts [7].

\section{Differential expression of IncRNAs, circRNAs and mRNAs upon osteogenic differentiation of PDLSCs}

Ribosome-depleted RNA-seq generated 91.4 million raw reads of the non-induced group and 113.8 million raw reads of the induced group (Table 1). After filtering the adaptor reads and low-quality tags, we separately obtained 90.9 million and 112.4 million clean reads. More than $98 \%$ of the raw reads per sample were clean reads. According to the annotation of the human genome sequence GENCODE Release 19, 11,529 lncRNAs (non-induced group) and 9511 lncRNAs (induced group) were identified from the two cDNA libraries (Table 2) (Additional file 2). We also identified 5913 circRNAs in the non-induced group and 3162 circRNAs in the induced group (Table 2) (Additional file 3). In addition, 66,134 mRNAs (noninduced group) and 52,227 mRNAs (induced group) 
were annotated (Table 2) (Additional file 4). Both lncRNAs and circRNAs were broadly distributed across the 24 pairs of human chromosomes (Fig. 2a, b). A total of 960 lncRNAs, 1161 circRNAs and 1887 mRNAs were found to be differentially expressed, with $P$-value $<0.05$ and $\mathrm{FC} \geq 2$ (Additional file 5, Additional file 6 and Additional file 7). Among the differentially expressed lncRNAs, 17 common lncRNAs and 180 specific lncRNAs in the non-induced group and 763 specific lncRNAs in the induced group were detected, with 777 up-regulated lncRNAs and 183 down-regulated lncRNAs (Fig. 2c). Meanwhile, we also identified 95 common circRNAs and 642 specific circRNAs in the non-induced group and 719 specific circRNAs in the induced group, with 766 upregulated circRNAs and 690 down-regulated circRNAs (Fig. 2d). The apparent variations in transcripts between the two groups are visually displayed with heatmaps (Fig. 2e, f).

\section{Differentially expressed IncRNAs/circRNAs validated by qRT-PCR}

To verify the results of the RNA-seq experiments, eight lncRNAs and eight circRNAs with $P<0.05, \mathrm{FC} \geq 2$ and
FPKM/RPM in at least one of the samples $\geq 4$ were selected for qRT-PCR validation. The lncRNAs were amplified with convergent primers and the circRNAs were amplified with divergent primers (Fig. 3a, b). Compared to the non-induced group, the induced group showed increased expression of the IncRNAs coded as TCONS_00019601, TCONS_00227764, TCONS_00254538, TCONS_00198784 and TCONS_00136898 and decreased expression of TCONS_00085268, TCONS_00125934, and TCONS_00 115113 (Fig. 3a). The circRNAs named CDR1-AS, NCOA3, and SKIL were up-regulated in the induced group compared to the non-induced group, and the circRNAs IFF01, NTNG1, PLOD2, SMO, and SMURF2 were down-regulated (Fig. 3b). All the results were consistent with the normalized expression of RNA-seq shown in Tables 2 and 3.

\section{Function of IncRNAs and circRNAs as ceRNAs via IncRNA/circRNA-miRNA-mRNA networks}

Based on miRanda with the maximum binding free energy of $-20,430$ lncRNAs were predicted to share at least two binding sites with 779 miRNAs (Fig. 4) (Additional file 8). We also predicted that 1401 circRNAs bind 855 miRNAs

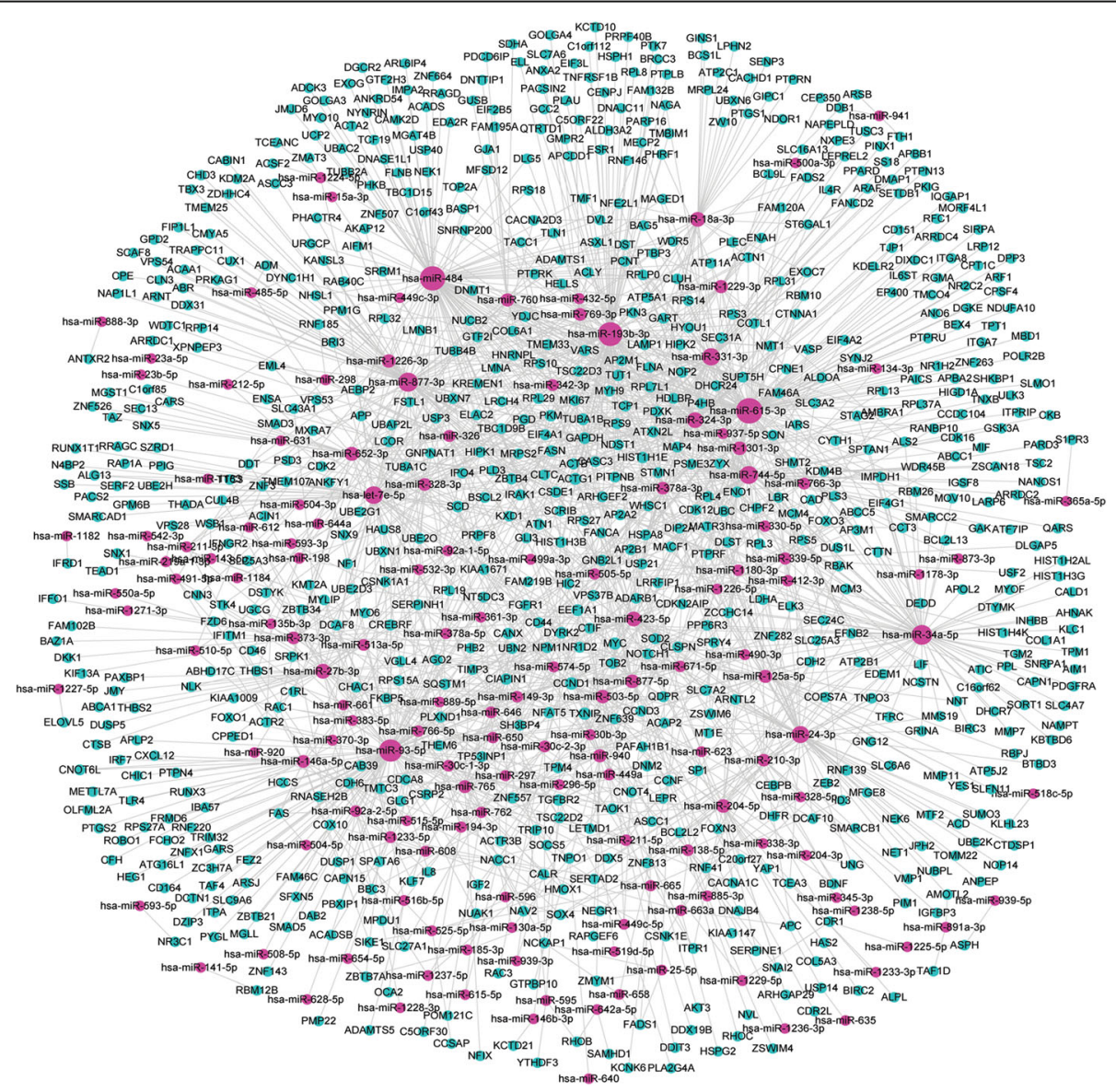

Fig. 6 CeRNA networks of 744 mRNAs and 148 common miRNAs based on miRanda 
with at least two binding sites (Additional file 9). Considering that graphics cannot display the enormous amount of network information between 1401 circRNAs and 855 miRNAs, we selected circRNAs with more miRNA binding sites and less binding free energy to make the network diagram (Fig. 5). Through analysis of the common binding MREs of lncRNAs and circRNAs, 165 miRNAs were predicted to combine with both 158 lncRNAs and 1385 circRNAs (data not shown).

To reveal their potential function, we predicted target mRNAs of these miRNAs based on miRanda and compared these target mRNAs to the differentially expressed mRNAs that were identified in the RNA-seq results (Additional file 7). There were 744 differentially expressed mRNAs that were found to combine with 148 common miRNAs along with 147 lncRNAs and 1382 circRNAs (Additional file 10). The networks of 744 mRNAs and 148 common miRNAs are shown in Fig. 6.

The potential regulatory roles of the ceRNA networks were predicted by analyzing the functions of 744 mRNAs through GO and KEGG pathway analysis (Additional file 11 and Additional file 12). GO annotations $(P<0.05)$ involving the top 60 mRNAs are displayed in Fig. 7a and include multiple biological processes, cellular components and molecular functions. Among these GO terms, we obtained GO: 0001649 (osteoblast differentiation), which was significantly enriched by 21 mRNAs (Additional file 11).

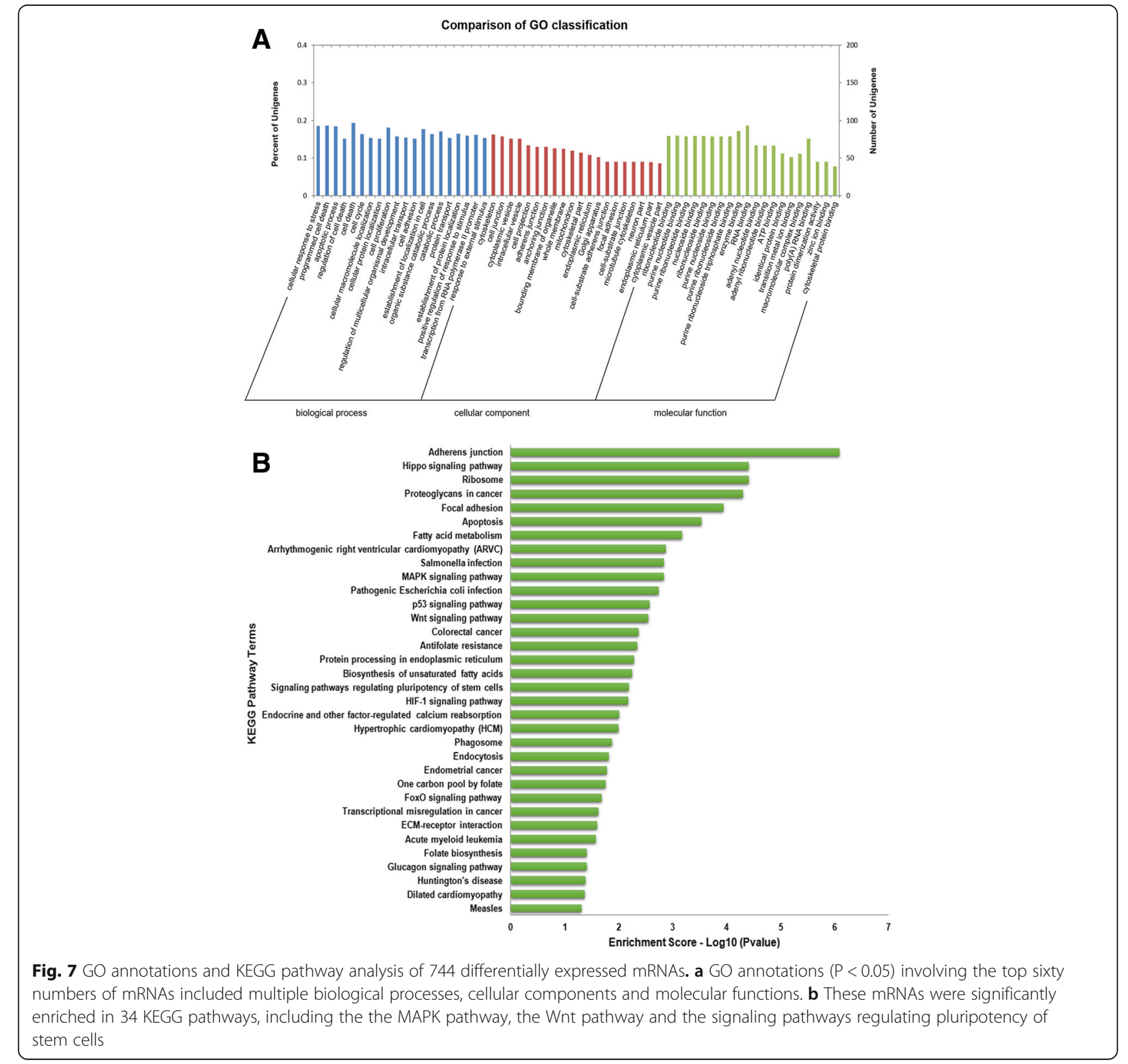


The complex mRNA networks involved in GO: 0001649 (osteoblast differentiation) and related miRNAs, lncRNAs, and circRNAs are displayed in Fig. 8. Through KEGG analysis, mRNAs were predicted to participte in 34 pathways (Fig. 7b). Among these KEGG pathways, the MAPK pathway, the Wnt pathway and the signaling pathways regulating pluripotency of stem cells were closely related to osteogenic differentiation.

Based on the above results, we selected several lncRNAs, circRNAs, miRNAs and mRNAs associated with the MAPK pathway to further display the ceRNA networks (Fig. 9). The lncRNAs coded as TCONS_00212979 and TCONS_00212984, circRNA BANP and circRNA ITCH were predicted to combine with miRNA34a and miRNA146a. DUSP1, FAS and RAC1 were predicted to be target genes of miRNA34a. In addition, PDGFRA, TGFBR2 and MYC were predicted to be targeted by miRNA146a. These six mRNAs were the pivotal genes of the MAPK pathway according to the KEGG analysis. This complicated ceRNA network suggested that TCONS_00212979, TCONS_00212984, circRNA BANP and circRNA ITCH might play regulatory roles in the MAPK pathway through miRNA34a, miRNA146a and their targets during PDLSC osteogenic differentiation.

\section{Discussion}

LncRNAs, circRNAs, miRNAs and mRNAs form largescale ceRNA cross-talk networks through MREs, which has exciting implications for gene regulation at the post-transcriptional level during multiple physiological and pathophysiological processes [12, 34]. In recent years, studies have documented the functions and clinical implications of ceRNAs in cancer, systemic lupus erythematosus and other diseases, which may present opportunities for new therapeutic approaches for diseases [35, 36].

Recently, researchers have systematically constructed ceRNA networks through RNA-seq and bioinformatics in mouse germline stem cells to reveal functions and mechanisms of lncRNAs and circRNAs in mouse germline stem cell self-renewal and differentiation [37]. Moreover, lncRNA POIR was demonstrated to form a regulatory network with miRNA182 and FoxO1 to up-regulate PDLSC osteogenic differentiation in periodontitis patients [38]. However, the ceRNA networks were revealed to be intertwined [39]. To fully understand the impact of ceRNA crosstalk on PDLSC osteogenic differentiation, it will be crucial to integrate the competitive lncRNA/ circRNA-miRNA-mRNA regulatory networks. In our study, 744 mRNAs were predicted to combine with 148

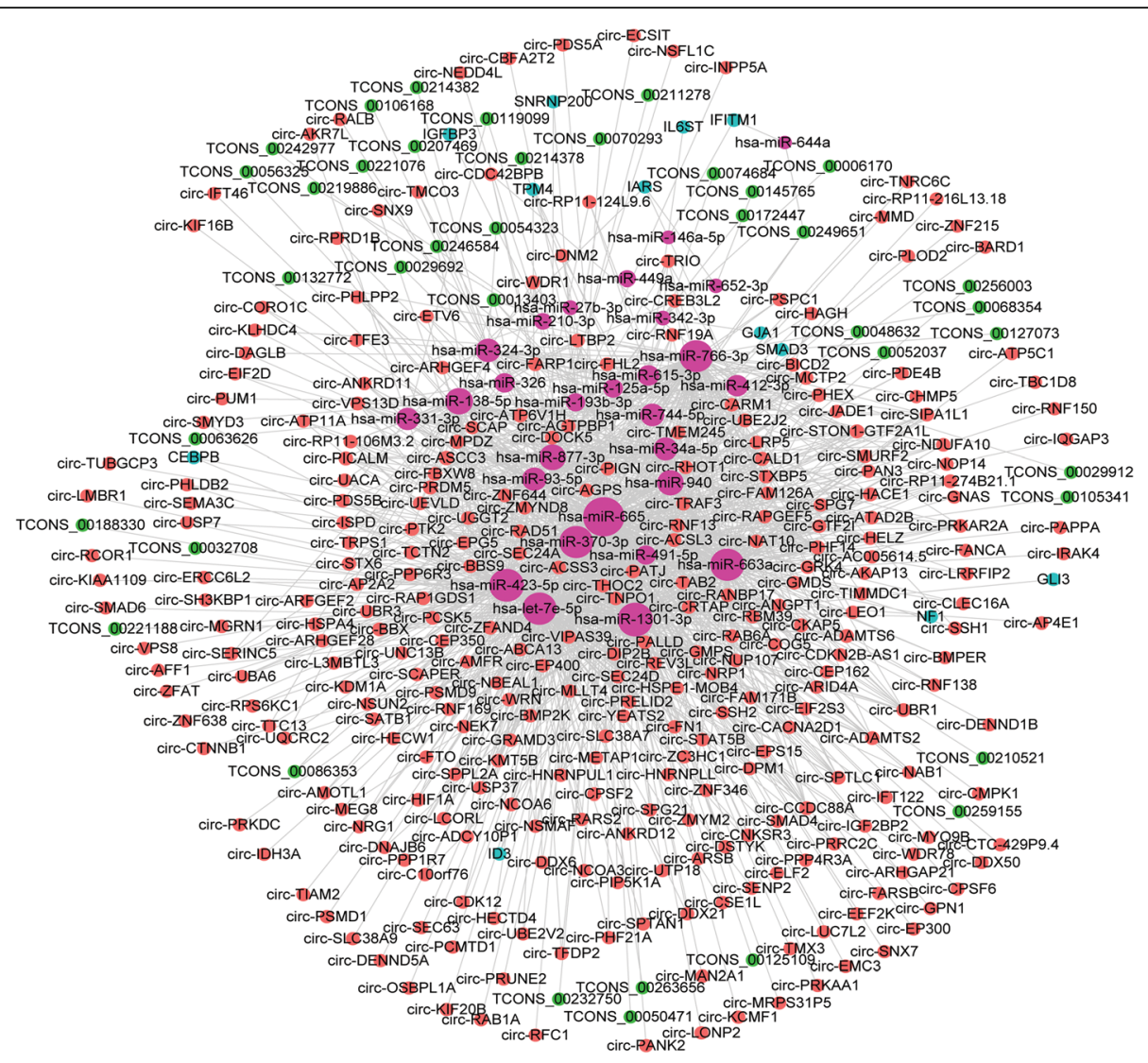

Fig. 8 CeRNA networks of IncRNAs/circRNAs-miRNAs-mRNAs significantly participated in GO: 0001649 (osteoblast differentiation) 


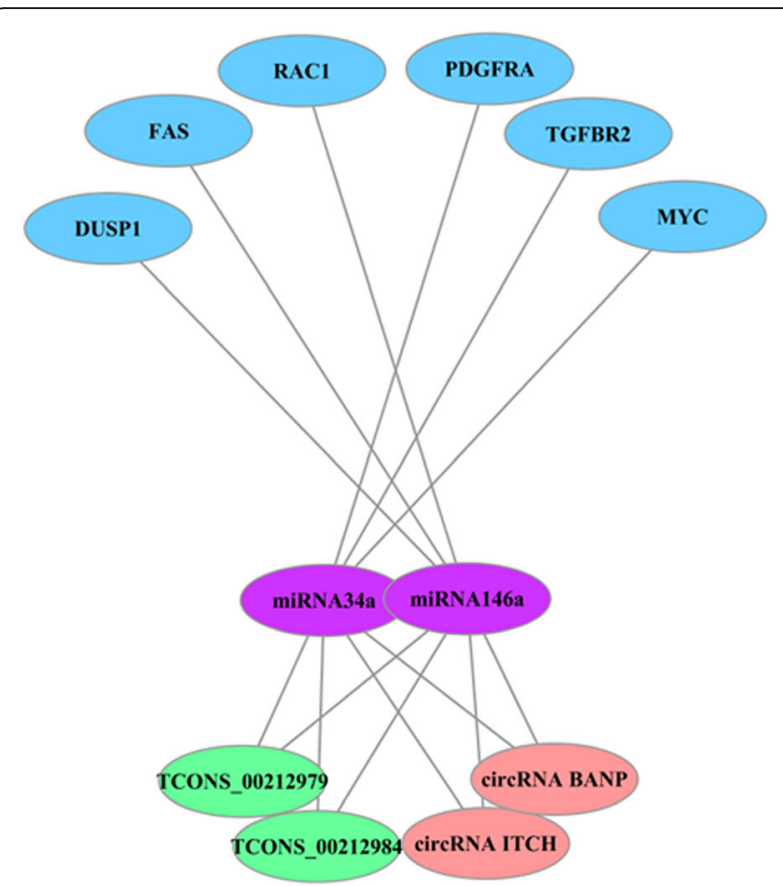

Fig. 9 CeRNA networks of IncRNAs/circRNAs-miRNAs-mRNAs significantly participated in the MAPK pathway. The IncRNAs coded as TCONS_00212979 and TCONS_00212984, as well as circRNA BANP and circRNA ITCH, were predicted to interact with miRNA34a and miRNA146a. DUSP1, FAS and RAC1 were predicted to be target genes of miRNA34a. PDGFRA, TGFBR2 and MYC were predicted to be targeted by miRNA146a. These six mRNAs are pivotal genes in the MAPK pathway according to KEGG analysis

common miRNAs, along with 147 lncRNAs and 1382 circRNAs.

Through GO analysis, 21 mRNAs were predicted to significantly participate in osteoblast differentiation (GO: 0001649) (Fig. 8). Among them, ALPL, also called ALP, was reported to be an osteogenesis-related marker and was up-regulated during PDLSC osteogenic differentiation [7]. The up-regulated expression level of ALP was also detected by qRT-PCR in our study (Fig. 1i). SMAD3 and SMAD5, members of the SMAD family, were also predicted to form ceRNA networks and participate in osteoblast differentiation. Both SMAD3 and SMAD5 were demonstrated to regulate PDLSC osteogenic differentiation by modulating TGF- $\beta$ signals $[40,41]$. Additionally, Notch1, another participant in osteoblast differentiation (GO: 0001649), is part of the Notch signaling pathway, which is important for maintaining osteogenic differentiation of PDLSCs [42, 43]. These crucial osteogenic genes formed ceRNA networks with lncRNAs and circRNAs by targeting common miRNAs, and these networks might provide evidence of new regulatory mechanisms in PDLSC osteogenic differentiation.
Through KEGG pathway analysis, mRNAs of ceRNA networks were predicted to be involved in the Wnt pathway, MAPK pathway and signaling pathways regulating the pluripotency of stem cells. Previous studies have demonstrated that Wnt signaling contributes to the differentiation of periodontal ligament fibroblasts into osteoblasts [44]. In addition, the MAPK pathway was found to be critical for skeleton development and bone homeostasis [45]. Moreover, it plays significant roles in osteogenic differentiation of PDLSCs [46, 47]. Furthermore, we constructed a ceRNA network of TCONS_00212979, TCONS 00212984, circRNA BANP, circRNA ITCH, miRNA34a, miRNA146a, DUSP1, FAS, RAC1, PDGFRA, TGFBR2 and MYC. These mRNAs are important elements of the MAPK pathway based on KEGG analysis. Among them, DUSP1, FAS and RAC1 are targeted by miRNA34a, while PDGFRA, TGFBR2 and MYC are targeted by miRNA146a. Studies have illustrated that both miRNA34a and miRNA146a are closely related to osteogenic differentiation of human mesenchymal stem cells [48, 49]. In addition, miRNA146a was revealed to promote differentiation of periodontal ligament cells [9]. TCONS_00212979, TCONS_00212984, circRNA BANP and circRNA ITCH were predicted to bind miRNA34a and miRNA146a. TCONS_00212979, known as CARMEN, has been reported to be a cardiac mesoderm enhancer-associated lncRNA that modulates cardiac differentiation through miRNA143 and miRNA145 [50]. TCONS_00212984 is a novel lncRNA with a genomic origin similar to that of TCONS_00212979 according to RNA-seq. circRNA BANP and circRNA ITCH have both been reported to contribute to carcinogenesis and might serve as cancer biomarkers [51, 52]. However, the regulatory roles of these two lncRNAs and circRNAs in osteogenic differentiation remain unclear. In summary, the ceRNA network suggested that TCONS_00212979, TCONS_00212984, circRNA BANP and circRNA ITCH might interact with miRNA34a and miRNA146a to regulate PDLSC osteogenic differentiation via the MAPK pathway. However, their regulatory mechanisms need to be further investigated. Our future study plan will be to validate their differential expression profiles, verify their ceRNA networks and specify their effects on PDLSC osteogenic differentiation using knockdown and overexpression experiments.

\section{Conclusion}

This study identified differentially expressed lncRNAs, circRNAs and mRNAs during osteogenic differentiation of PDLSCs. Competitive IncRNA/circRNA-miRNA-mRNA regulatory networks were comprehensively integrated and predicted to be involved in osteoblast differentiation by GO and KEGG pathway analysis. Moreover, the lncRNAs coded as TCONS_00212979 and TCONS_00212984, circRNA BANP and circRNA ITCH were predicted to 
interact with miRNA34a and miRNA146a to regulate PDLSC osteogenic differentiation via the MAPK pathway. Our study suggested that specific lncRNAs and circRNAs might function as ceRNAs to promote PDLSC osteogenic differentiation and periodontal regeneration.

\section{Additional files}

Additional file 1: Gene Primers.doc Gene primers used in qRT-PCR (DOC $38 \mathrm{~kb}$ )

Additional file 2: IncRNA_all expression_.xlsx The expression profiles of all IncRNAs identified by RNA-seq (XLSX $1653 \mathrm{~kb}$ )

Additional file 3: circRNA_all expression_.xlsx The expression profiles of all circRNAs identified by RNA-seq (XLSX $1577 \mathrm{~kb}$ )

Additional file 4: $m R N A \_a l l$ expression_.xlsx The expression profiles of all mRNAs identified by RNA-seq (XLSX $8078 \mathrm{~kb}$ )

Additional file 5: IncRNA_differential expression.xlsx Differentially expressed IncRNAs identified by RNA-seq (XLSX $87 \mathrm{~kb}$ )

Additional file 6: circRNA_differential expression.xlsx Differentially expressed circRNAs identified by RNA-seq (XLSX $312 \mathrm{~kb}$ )

Additional file 7: mRNA_differential expression.xlsx Differentially expressed mRNAs identified by RNA-seq (XLSX $240 \mathrm{~kb}$ )

Additional file 8: IncRNA_miRNA_miRanda_2BindingSites.xlsx LncRNAs predicted to share at least two miRNA binding sites (XLSX $115 \mathrm{~kb}$ )

Additional file 9: circRNA_miRNA_miRanda_2BindingSites.xlsx CircRNAs predicted to share at least two miRNA binding sites (XLSX $23183 \mathrm{~kb}$ )

Additional file 10: IncRNA-circRNA-miRNA-mRNA.xlsx LncRNAs and circRNAs predicted to share miRNA binding sites in common with target mRNAs (XLSX $8696 \mathrm{~kb}$ )

Additional file 11: target mRNAs_GO enrichment.xlsX GO items enriched by target mRNAs through GO analysis (XLSX $95 \mathrm{~kb}$ )

Additional file 12: target mRNAs_KEGG pathway enrichment.xlsX Pathways enriched by target mRNAs through KEGG analysis (XLSX $20 \mathrm{~kb}$ )

\section{Abbreviations}

ALP: Alkaline phosphatase; bp: Base pairs; ceRNAs: Competitive endogenous RNAs; circRNAs: Circular RNAs; FC: Fold change; FPKM: Fragments per kilobase of exon per million fragments mapped; GO: Gene Ontology; KEGG: Kyoto Encyclopedia of Genes and Genomes; IncRNAs: Long noncoding RNAs; miRNAs: MicroRNAs; MREs: miRNA response elements; mRNAs: Messenger RNAs; ncRNA: Non-coding RNA; OCN: Osteocalcin; PDLSC: Periodontal ligament stem cell; qRT-PCR: Quantitative real-time polymerase chain reaction; RNA-seq: RNA sequencing; RPM: Mapped back-splice junction reads per million mapped reads; Runx2: Runt-related transcription factor 2; SD: Standard deviation

\section{Acknowledgments}

We acknowledge the anonymous reviewers and academic editors for their constructive suggestions on the previous version of the manuscript. We acknowledge laboratory members for their contributions.

\section{Funding}

The performance of RNA-seq, analysis of the sequenced data and revision of the manuscript were supported by grant from the National Natural Science Foundation of China (81470709). All the cell biology experiments and molecular biology experiments were supported by grant from the Construction Engineering Special Fund of "Taishan Scholars" (tsqn201611068).

\section{Availability of data and materials}

All data analyzed during this study are included in this article and its supplementary information files. RNA-seq data sets are available in the Gene Expression Omnibus database under accession number GSM2856840 (for non-induced PDLSCs) and GSM2856841 (for induced PDLSCs).

\section{Authors' contributions}

FW and DL conceived, designed and revised this study. XG analyzed the RNA-seq data and wrote the manuscript. ML detected the expression of genes using qRT-PCR. YJ performed the cell culture and osteogenic induction treatments. All authors read and approved the final manuscript.

Ethics approval and consent to participate

PDLSCs were derived from the extracted teeth, which were collected from patients at the Department of Oral Maxillofacial Surgery, Stomatology Hospital of Shandong University. All protocols for handling dental tissues were performed in accordance with relevant guidelines and regulations. All protocols for the handling of human tissues were approved by the Research Ethics Committee of Stomatology Hospital of Shandong University, China (G201401601). The participant informed consent was written.

\section{Consent for publication}

Not applicable.

\section{Competing interests}

The authors declare that they have no competing interests.

\section{Publisher's Note}

Springer Nature remains neutral with regard to jurisdictional claims in published maps and institutional affiliations.

\section{Author details}

'Department of Orthodontics, Shandong Provincial Key Laboratory of Oral Tissue Regeneration, School of Stomatology, Shandong University, Wenhua Xi Road No. 44-1, Jinan, Shandong 250012, People's Republic of China.

${ }^{2}$ Shandong Provincial Key Laboratory of Oral Tissue Regeneration, School of Stomatology, Shandong University, Jinan, People's Republic of China.

Received: 19 August 2017 Accepted: 16 November 2017

Published online: 02 December 2017

\section{References}

1. Damgaard C, Holmstrup P, Van Dyke TE, Nielsen CH. The complement system and its role in the pathogenesis of periodontitis: current concepts. J Periodontal Res. 2015:50(3):283-93.

2. Liu Y, Zheng Y, Ding G, Fang D, Zhang C, Bartold PM, Gronthos S, Shi S, Wang S. Periodontal ligament stem cell-mediated treatment for periodontitis in miniature swine. Stem Cells. 2008;26(4):1065-73.

3. Park JY, Jeon SH, Choung PH. Efficacy of periodontal stem cell transplantation in the treatment of advanced periodontitis. Cell Transplant. 2011;20(2):271-85

4. Wei F, Qu C, Song T, Ding G, Fan Z, Liu D, Liu Y, Zhang C, Shi S, Wang S, Vitamin C. Treatment promotes mesenchymal stem cell sheet formation and tissue regeneration by elevating telomerase activity. J Cell Physiol. 2012; 227(9):3216-24.

5. Wei F, Song T, Ding G, Xu J, Liu Y, Liu D, Fan Z, Zhang C, Shi S, Wang S. Functional tooth restoration by allogeneic mesenchymal stem cell-based bio-root regeneration in swine. Stem Cells Dev. 2013;22(12):1752-62.

6. Ding G, Liu Y, Wang W, Wei F, Liu D, Fan Z, An Y, Zhang C, Wang S. Allogeneic periodontal ligament stem cell therapy for periodontitis in swine. Stem Cells. 2010;28(10):1829-38.

7. Seo BM, Miura M, Gronthos S, Bartold PM, Batouli S, Brahim J, Young M, Robey PG, Wang CY, Shi S. Investigation of multipotent postnatal stem cells from human periodontal ligament. Lancet. 2004;364(9429):149-55.

8. Hao Y, Ge Y, Li J, Hu Y, Wu B, Fang F. Identification of MicroRNAs by microarray analysis and prediction of target genes involved in the Osteogenic differentiation of human periodontal ligament stem cells. J Periodontol. 2017:1-11.

9. Hung PS, Chen FC, Kuang SH, Kao SY, Lin SC, Chang KW. miR-146a induces differentiation of periodontal ligament cells. J Dent Res. 2010;89(3):252-7.

10. Liu Y, Liu W, Hu C, Xue Z, Wang G, Ding B, Luo H, Tang L, Kong X, Chen X, et al. MiR-17 modulates osteogenic differentiation through a coherent feedforward loop in mesenchymal stem cells isolated from periodontal ligaments of patients with periodontitis. Stem Cells. 2011;29(11):1804-16.

11. Yan GQ, Wang X, Yang F, Yang ML, Zhang GR, Wang GK. MicroRNA-22 promoted Osteogenic differentiation of human periodontal ligament stem cells by targeting HDAC6. J Cell Biochem. 2017;118(7):1653-8. 
12. Salmena $L$, Poliseno $L$, Tay $Y$, Kats $L$, Pandolfi PP. A ceRNA hypothesis: the Rosetta stone of a hidden RNA language? Cell. 2011;146(3):353-8.

13. Sen R, Ghosal S, Das S, Balti S, Chakrabarti J. Competing endogenous RNA: the key to posttranscriptional regulation. TheScientificWorldJOURNAL. 2014; 2014:896206.

14. Kapranov $P$, Cheng J, Dike S, Nix DA, Duttagupta R, Willingham AT, Stadler PF, Hertel J, Hackermuller J, Hofacker IL, et al. RNA maps reveal new RNA classes and a possible function for pervasive transcription. Science. 2007; 316(5830):1484-8

15. Fatica A, Bozzoni I. Long non-coding RNAs: new players in cell differentiation and development. Nat Rev Genet. 2014;15(1):7-21.

16. WS XY, Tang C, Chen W. Upregulation of long non-coding RNA HIF 1a-antisense 1 induced by transforming growth factor- $\beta$-mediated targeting of sirtuin 1 promotes osteoblastic differentiation of human bone marrow stromal cells. Mol Med Rep. 2015;12(5):7233-8

17. Jia $Q$, Jiang W, Ni L. Down-regulated non-coding RNA (IncRNA-ANCR) promotes osteogenic differentiation of periodontal ligament stem cells. Arch Oral Biol. 2015;60(2):234-41.

18. Wilusz JE, Sharp PA. Molecular biology. A circuitous route to noncoding RNA. Science. 2013;340(6131):440-1.

19. Qu S, Yang X, Li X, Wang J, Gao Y, Shang R, Sun W, Dou K, Li H. Circular RNA: a new star of noncoding RNAs. Cancer Lett. 2015;365(2):141-8.

20. Barrett SP, Salzman J. Circular RNAs: analysis, expression and potential functions. Development. 2016;143(11):1838-47.

21. Lasda E, Parker R. Circular RNAs: diversity of form and function. RNA. 2014; 20(12):1829-42.

22. Qian DY, Yan GB, Bai B, Chen Y, Zhang SJ, Yao YC, Xia H. Differential circRNA expression profiles during the BMP2-induced osteogenic differentiation of MC3T3-E1 cells. Biomedicine \& pharmacotherapy = Biomedecine \& pharmacotherapie. 2017:90:492-9.

23. Zheng Y, Li X, Huang Y, Jia L, Li W. The circular RNA landscape of periodontal ligament stem cells during Osteogenesis. J Periodontol. 2017:88(9):906-14.

24. Wei FL, Wang JH, Ding G, Yang SY, Li Y, YJ H, Wang SL. Mechanical forceinduced specific MicroRNA expression in human periodontal ligament stem cells. Cells Tissues Organs. 2014;199(5-6):353-63.

25. Borodina T, Adjaye J, Sultan M. A strand-specific library preparation protocol for RNA sequencing. Methods Enzymol. 2011;500:79-98.

26. Kim D, Pertea G, Trapnell C, Pimentel H, Kelley R, Salzberg SL. TopHat2: accurate alignment of transcriptomes in the presence of insertions, deletions and gene fusions. Genome Biol. 2013;14(4):R36.

27. Trapnell C, Williams BA, Pertea G, Mortazavi A, Kwan G, van Baren MJ, Salzberg SL, Wold BJ, Pachter L. Transcript assembly and quantification by RNA-Seq reveals unannotated transcripts and isoform switching during cell differentiation. Nat Biotechnol. 2010;28(5):511-5.

28. Kong L, Zhang Y, Ye ZQ, Liu XQ, Zhao SQ, Wei L, Gao G. CPC: assess the protein-coding potential of transcripts using sequence features and support vector machine. Nucleic Acids Res. 2007:35(Web Server issue):W345-9.

29. Anders $S$, Huber W. Differential expression analysis for sequence count data. Genome Biol. 2010;11(10):R106.

30. Zhang XO, Dong R, Zhang Y, Zhang JL, Luo Z, Zhang J, Chen LL, Yang L. Diverse alternative back-splicing and alternative splicing landscape of circular RNAs. Genome Res. 2016;26(9):1277-87.

31. Leng N, Dawson JA, Thomson JA, Ruotti V, Rissman Al, Smits BM, Haag JD, Gould MN, Stewart RM, Kendziorski C. EBSeq: an empirical Bayes hierarchical model for inference in RNA-seq experiments. Bioinformatics. 2013;29(8):1035-43.

32. miRanda: http://www.microrna.org/microrna/home.do Assessed August 2010.

33. Conesa A, Gotz S, Garcia-Gomez JM, Terol J, Talon M, Robles M. Blast2GO: a universal tool for annotation, visualization and analysis in functional genomics research. Bioinformatics. 2005;21(18):3674-6.

34. Ala U, Karreth FA, Bosia C, Pagnani A, Taulli R, Leopold V, Tay Y, Provero P, Zecchina R, Pandolfi PP. Integrated transcriptional and competitive endogenous RNA networks are cross-regulated in permissive molecular environments. Proc Natl Acad Sci U S A. 2013;110(18):7154-9.

35. Qi X, Zhang D-H, Wu N, Xiao J-H, Wang X, Ma W. ceRNA in cancer: possible functions and clinical implications. J Med Genet. 2015;52(10):710-8.

36. Li LJ, Zhao W, Tao SS, Leng RX, Fan YG, Pan HF, Ye DQ. Competitive endogenous RNA network: potential implication for systemic lupus erythematosus. Expert Opin Ther Targets. 2017;21(6):639-48.

37. Li X, Ao J, Wu J. Systematic identification and comparison of expressed profiles of IncRNAs and circRNAs with associated co-expression and ceRNA networks in mouse germline stem cells. Oncotarget. 2017;8(16):26573-90.
38. Wang L, Wu F, Song Y, Li X, Wu Q, Duan Y, Jin Z. Long noncoding RNA related to periodontitis interacts with miR-182 to upregulate osteogenic differentiation in periodontal mesenchymal stem cells of periodontitis patients. Cell Death Dis. 2016;7(8):e2327.

39. Tay Y, Rinn J, Pandolfi PP. The multilayered complexity of ceRNA crosstalk and competition. Nature. 2014;505(7483):344-52.

40. Huang Y, Zheng Y, Jia L, Li W. Long noncoding RNA H19 promotes Osteoblast differentiation via TGF-beta1/Smad3/HDAC signaling pathway by deriving miR-675. Stem Cells. 2015;33(12):3481-92.

41. Ishibashi O, Ikegame M, Takizawa F, Yoshizawa T, Moksed MA, lizawa F, Mera H, Matsuda A, Kawashima H: Endoglin is involved in BMP-2-induced osteogenic differentiation of periodontal ligament cells through a pathway independent of Smad-1/5/8 phosphorylation. J Cell Physiol 2010, 222(2):465-473.

42. Osathanon T, Ritprajak P, Nowwarote N, Manokawinchoke J, Giachelli C, Pavasant P. Surface-bound orientated Jagged-1 enhances osteogenic differentiation of human periodontal ligament-derived mesenchymal stem cells. J Biomed Mater Res A. 2013;101(2):358-67.

43. Li Y, Li SQ, Gao YM, Li J, Zhang B. Crucial role of notch signaling in osteogenic differentiation of periodontal ligament stem cells in osteoporotic rats. Cell Biol Int. 2014;38(6):729-36.

44. Heo JS, Lee SY, Lee JC. Wnt/beta-catenin signaling enhances osteoblastogenic differentiation from human periodontal ligament fibroblasts. Molecules and cells. 2010;30(5):449-54.

45. Thouverey C, Caverzasio J. Focus on the p38 MAPK signaling pathway in bone development and maintenance. BoneKEy reports. 2015;4:711.

46. Tang M, Peng Z, Mai Z, Chen L, Mao Q, Chen Z, Chen Q, Liu L, Wang Y, Ai $H$. Fluid shear stress stimulates osteogenic differentiation of human periodontal ligament cells via the extracellular signal-regulated kinase 1/2 and p38 mitogen-activated protein kinase signaling pathways. J Periodontol. 2014;85(12):1806-13.

47. Li L, Han MX, Li S, Xu Y, Wang L. Hypoxia regulates the proliferation and osteogenic differentiation of human periodontal ligament cells under cyclic tensile stress via mitogen-activated protein kinase pathways. J Periodontol. 2014;85(3):498-508.

48. Chen L, Holmstrom K, Qiu W, Ditzel N, Shi K, Hokland L, Kassem M. MicroRNA-34a inhibits osteoblast differentiation and in vivo bone formation of human stromal stem cells. Stem Cells. 2014:32(4):902-12.

49. Huszar JM, Payne CJ. MIR146A inhibits JMJD3 expression and osteogenic differentiation in human mesenchymal stem cells. FEBS Lett. 2014;588(9):1850-6.

50. Ounzain S, Micheletti R, Arnan C, Plaisance I, Cecchi D, Schroen B, Reverter F, Alexanian M, Gonzales C, Ng SY, et al. CARMEN, a human super enhancerassociated long noncoding RNA controlling cardiac specification, differentiation and homeostasis. J Mol Cell Cardiol. 2015;89(Pt A):98-112.

51. Zhu M, Xu Y, Chen Y, Yan F, Circular BANP. An upregulated circular RNA that modulates cell proliferation in colorectal cancer. Biomedicine \& pharmacotherapy $=$ Biomedecine \& pharmacotherapie. 2017:88:138-44.

52. Guo W, Zhang J, Zhang D, Cao S, Li G, Zhang S, Wang Z, Wen P, Yang H, Shi $X$, et al. Polymorphisms and expression pattern of circular RNA circ-ITCH contributes to the carcinogenesis of hepatocellular carcinoma. Oncotarget. 2017:8(29):48169-77.

\section{Submit your next manuscript to BioMed Central and we will help you at every step:}

- We accept pre-submission inquiries

- Our selector tool helps you to find the most relevant journal

- We provide round the clock customer support

- Convenient online submission

- Thorough peer review

- Inclusion in PubMed and all major indexing services

- Maximum visibility for your research

Submit your manuscript at www.biomedcentral.com/submit 